\title{
Lost in Translation. Translating the Latin Words of the Pirandello's Novels
}

\author{
Prof. As. Dr. Mirela Papa \\ Docente di Traduzione e Interpretariato, Università di Tirana \\ papamirela@yahoo.com \\ Dr. Leonard Xhamani \\ Docente di Latino, Università di Tirana \\ leonardxhemani@hotmail.com
}

Doi:10.5901/jesr.2014.v4n4p361

\begin{abstract}
The translator is an interlinguistic and intercultural mediator. In the process of translation he is like a bridge between two groups of people who don't share the same linguistic and cultural code and as a result they use the translator in order to communicate between them. Nowadays it is a fact in the theory of communication that in every kind of communication there is loss during the process. With loss we mean that element of the message that is not conveyable. We encounter this loss even in translation. With loss, in the process of translation, we mean that element of translation, which the translator after planning his translation strategy, decides not to translate it because it is very difficult or even impossible to be translated. In this paper we will study the Latin words that the Italian writer Luigi Pirandello uses in that the Italian writer Luigi Pirandello use in his novels. The target of this analysis will be the strategy that the Albanian translators have used in order to provide these textual segments in the novels translated in the Albanian language. Taking into consideration that the Albanian and Italian language differ a lot among them we will notices that during the translation process we will encounter inevitable loss.
\end{abstract}

Keywords: fiction, Latin words, loss, prose, translation.

\section{Introduzione allo Studio}

La lingua di Pirandello sembra una lingua facile che non pone alcun problema per i traduttori. Essendo contro i dettami della stilistica dell'epoca Pirandello elabora una lingua che assomiglia assai alla lingua parlata. Eppure anche in questa lingua apparentemente così semplice il traduttore si imbatte in difficoltà e deve elaborare delle strategie per poterle superare.

In questo studio prenderemo in analisi i latinismi usati dallo scrittore italiano Luigi Pirandello nei suoi romanzi. Oggetto di analisi sarà la strategia seguita da parte dei traduttori albanesi per dare questi segmenti testuali nei romanzi tradotti in albanese.

II corpus dello studio viene costituito pertanto da:

1999, L'esclusa (Marta Ajala), traduttore Zija Vukaj, Tirana: Elite.

2000, Il fu Mattia Pascal (Tre herë vetëvrasës), traduttore Sabah Ramja, Shkodër: Helena Kadare.

2001, Uno, nessuno e centomila (Një, asnjë dhe njëqind mijë), traduttore Zija Vukaj, Tirana: Elite.

2002, Uno, nessuno e centomila (Një, asnjë dhe njëqind mijë), traduttore Dritan Çela, Elbasan: Sejko.

2003, II fu Mattia Pascal (I ndjeri Matia Paskal), traduttrice Violanda Canko, Tirana: Elena Gjika.

\section{Perdite nella Traduzione}

Tradurre vuole dire capire il sistema interno di una lingua e la struttura di un testo dato in quella lingua, e costruire un doppio del sistema testuale che, sotto una certa descrizione, possa produrre effetti analoghi nel lettore, sia sul piano semantico e sintattico che su quello stilistico, metrico, fonosimbolico, e quanto agli effetti passionali a cui il testo fonte tendeva. "Sotto una certa descrizione" significa che ogni traduzione presenta dei margini di infedeltà rispetto a un nucleo di presunta fedeltà, ma la decisione circa la posizione del nucleo e l'ampiezza dei margini dipende dai fini che si pone il traduttore (Eco, 2003: 16-17). Dunque tocca al traduttore esaminare i vari piani costituenti un'opera letteraria e determinare quali saranno i piani da trasportare nella lingua d'arrivo ed il grado di trasposizione. Naturalmente sarà compito arduo per il traduttore cercare di dare tutti i piani, perciò ci saranno degli elementi che si trasporteranno nella LA e ci saranno altri elementi che andranno persi nell'impossibilità di essere dati. Come sostiene G. Mounin, la teoria deve 
prendere atto che effettivamente la traduzione non è sempre del tutto possibile.

Susan Bassnet scrive (1993: 48) che una volta accettato il principio che non può esistere uguaglianza fra due lingue, diventa possibile parlare di perdite e delle acquisizioni nel processo della traduzione. Bruno Osimo nel suo Manuale del traduttore (2004: 222) dà questa definizione della perdita. Residuo: nella teoria della matematica della comunicazione, elemento del messaggio che non giunge a destinazione. Perdita. Elemento della traduzione che, dopo avere elaborato la propria strategia, il traduttore decide di non tradurre all'interno del testo nella cultura ricevente perché risulta una delle sottodominanti meno prioritarie 0 , comunque, risulta difficile 0 apparentemente impossibile da tradurre. $\mathrm{Ci}$ sono delle perdite che potremmo definire assolute. Sono i casi in cui non è possibile tradurre. Ci sono casi in cui la perdita, se ci si attiene alla lettera del testo, è irrimediabile (Osimo, 2004: 95).

\section{I Latinismi nei Romanzi di Pirandello}

«Oltre che in forma dell'elemento latino autoctono, il latino è presente nelle lingue da esso discendenti anche in un altro modo, 0 in un'altra forma: esso, cioè, anche nei secoli posteriori all'Alto Medioevo (...) rappresenta una delle fonti più importanti di tutte. (Tekavcic citato da Navarro Blanco, 1990: 66)» Le lingue romanze si sono sviluppate, come si sa, a partire dal latino parlato e si sono evolute fino ad essere lingue distinte rispetto al latino. Riescono comunque a mantenere un contatto diretto non solo nella prima epoca della loro vita, ma anche più tardi quando l'esistenza di queste lingue era innegabile ormai. «I contatti delle lingue romanze nascenti e del latino non si arrestano a questa prima fase: durante tutto il periodo di vita delle lingue neolatine, fino ai nostri giorni, i vocaboli esprimenti concetti astratti, scientifici, filosofici, artistici ecc. vengono presi dal latino o formati con elementi latini... (Navarro Blanco, 1990: 67)»

Nella lingua italiana i prestiti provenienti direttamente dal latino si conoscono con il nome «termini colti», anche se si possono chiamare anche latinismi. Si distinguono dall'altra parte del lessico autoctono da una serie di criteri fonetici e semantici. Indipendetemente da queste differenze, le voci colte (o dotte) fanno parte del lessico italiano esattamente come le voci «popolari».

Ritornando al Pirandello, bisogna dire che i latinismi costituiscono un elemento essenziale del suo lessico, come succedeva del resto anche negli altri scrittori come Carducci, D'Annunzio e Pascoli. Lo studio e poi dopo l'influenza della lingua e della letteratura latina ha influenzato molto lo stile e la lingua di Luigi Pirandello, che sembra sempre permeata da vari e numerosi cultismi (Papa \& Xhamani, 2013: 237). In Pirandello si distinguono latinismi integrati in italiano (cioè italianizzati, adattati alla struttura fono-morfologica delle parole italiane) i quali conservano la qualità dei cultismi indicati spesso come termini letterari e poetici, e latinismi non integrati (mantenendo inalterata la loro forma di voci latine) che l'autore li usa come espressioni lessicalizzate nella lingua e in alcuni altri casi come litania non senza un pizzico di ironia e critica. (Navarro Blanco, 1990: 68)

\subsection{Esempi tratti dal romanzo II fu Mattia Pascal.}

\subsubsection{Latinismi non integrati}

$>$ (sit venia verbo)

- $\quad$ Si omette nella traduzione. (RAMJA, V, 42)

- $\quad$ Si riproduce letteralmente nel testo tradotto. (CANKO, V, 40)

$>$ Mea mihi conscientia pluris est quam hominum sermo.

- $\quad$ Si riproduce letteralmente nel testo tradotto (RAMJA, IX, 101)

- Si riproduce letteralmente nel testo tradotto (CANKO, IX, 95)

$>$ In questo caso, - gli feci osservare, - mi sembra che più di tutti, scusa, dovrei risentirmi io, che vedrò d'ora innanzi la mia bella quondam metà convivere maritalmente con te.

- Më falni, por më duket, se në këtë rast, më tepër se të gjithë e ndjej unë që do të shoh këtej e tutje bashkëshorten time quondam e të bukur të bashkëjetojë me ty. (glossa extratestuale) të dikurshme. (RAMJA, $\mathrm{XVIII,} \mathrm{235)}$

- Në këtë rast, - i tërheq vëmendjen unë, - më fal, po më duket se jam unë për të qarë hallin, unë që këtej e tutje do ta shoh gjysmën time bukuroshe të bashkëjetojë me ty. (CANKO, XVIII, 222)

$>$ Vale, diletto amico, vale! (Una formula di saluto in latino)

- Ja vlen, mik i zgjedhur ja vlen. (RAMJA, VII, 80)

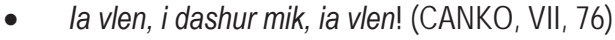


Per quanto riguarda la strategia traduttiva per i segmenti testuali in latino, predomina la strategia della ripetizione, seguita da quella dell'omissione intera del termine e la spiegazione con una glossa extratestuale.

\subsubsection{Latinismi integrati}

$>E$ io? che potevo far io? Denunziarlo?

- Po unë? Çfarë mund të bëja? Ta kallëzoja në polici? (RAMJA, XV, 186)

- Po unë? Ç'mund të bëj unë? Ta akuzoj? (CANKO, XV, 175)

$>$ È vero che due di essi mi s'avventarono contro anche coi coltelli.

- Dy nga ata m'u vërtitën me thika në dorë. (RAMJA, XI, 123)

- Vërtet që dy syresh m'u vërsulën edhe me thika. (CANKO, XI, 117)

$>$ E dunque, doveva ella credere che fosse generosità da parte mia, sacrifizio per amor di lei?

- A duhet të besonte ajo, se kjo ishte zemërgjerësi e imja, sakrificë për dashurinë e saj? (RAMJA, XVI, 196)

- E kështu ajo do të pandehte se ishte një qëndrim bujar nga ana ime, një sakrificë që e bëja për hir të dashurisë për të? (CANKO, XVI, 185)

$>$ lo ora vivo in pace, insieme con la mia vecchia zia Scolastica, che mi ha voluto offrir ricetto in casa sua.

- Unë tani jetoj në qetësi, bashkë me hallë Skolastikën, e cila më ka strehuar në shtëpinë e saj. (RAMJA, XVIII, 239)

- Tani jetoj në paqe me teton time të moshuar Skolastika, që deshi e më strehoi në shtëpisë e saj. (CANKO, $X V I I I, 227)$

> Una magnifica parrucca fulva, riccioluta, e - su la parrucca - un ampio fazzoletto di seta cilestrina, anzi uno scialle, annodato artisticamente sotto il mento.

- Një paruke madhështore kuqalashe, me kaçurrela dhe mbi paruke kishte një shami mëndafshi të kaltëremë, bile kishte edhe një shall të lidhur bukur në gushë. (RAMJA, XVI, 201)

- Një parrukë kuqalashe, kaçurrelse dhe, mbi parrukë - një shami e madhe mëndafshi, e kaltëreme, madje më mirë të themi, një shall, i lidhur mjeshtërisht në gushë. (CANKO, XVI, 189)

$>$ «l doni del Lama?» domandai a me stesso, chiudendo gli occhi, cogitabondo. «Che saranno?»

- Dhuratat e Lamës? - pyeta veten duke mbyllur sytë i menduar. Ç'të kenë qenë vallë? (RAMJA, VII, 73)

- Dhuratat e Lamës? - pyeta vehten duke mbyllur sytë i menduar. Çfarë do të jenë? (CANKO, VII, 69)

$>$ A MONSIGNOR BOCCAMAZZA

MUNIFICENTISSIMO DONATORE

IN PERENNE ATTESTATO DI GRATITUDINE

I CONCITTADINI

QUESTA LAPIDE POSERO

- MONSINJOR BOKAMACËS

DHURUES SHUMË BUJAR

SI DËSHMI E PËRHERSHME MIRËNJOHJEJE

BASHKËQYTETARËT

I VUNË KËTË KUJTIM. (RAMJA, V, 46)

- KUSHTUAR

MONSINJOR BOKAMACËS

QË I LA LIBRAT TRASHËGIM BASHKISË

NË SHENJË MIRËNJOHJEJE TË PËRJETSHME

KËTË LAPIDAR I NGREJNË

BASHKËQYTETARËT (CANKO, V, 44)

$>$ Improvvisamente, mi vidi in una condizione assai speciosa.

- Papritmas e pashë veten në një gjendje mjaft të veçantë. (RAMJA, $X, 114$ )

- Papritur e pakujtuar e ndiej veten në një gjendje tepër të paqartë. (CANKO, $X, 107)$

> Ecco: essa, per esempio, voleva dire starmene lì, di sera, affacciato a una finestra, a guardare il fiume ch e fluiva nero e silente tra gli argini nuovi e sotto i ponti che vi riflettevano i lumi dei loro fanali, tremolanti come serpentelli di fuoco;

- Ja! Për shembull, ajo donte të më thoshte që të qëndroja aty në mbrëmje, në dritare duke shikuar lumin, që 
rridhte i zi e i heshtur, mes pritave të reja dhe urave, që pasqyronin dritat e fenerëve të tyre, të dridheshin si gjarpërinj të zjarrtë. (RAMJA, XI, 120)

- Dhe ja: liria, që gëzoja, për shembull, më pëshpëriste në vesh të mos guxoja e të nxirrja kokën jashtë mbrëmjeve e të rrija urtë, pështetur pas xhamit të dritares, për të kundruar lumin, i cili rridhte i zi dhe i heshtur përmes pendave të sapongritura dhe nën urat, duke pasqyruar dritën e fenerëve të tyre, drithëruese si ca gjarpërinj të zjarrtë; (CANKO, XI, 114)

$>$ Con undicimila lire avrei potuto rimetter la pace in casa e far rinascere l'amore già iniquamente ucciso in sul nascere dalla vedova Pescatore?

- Me njëmbëdhjetë mijë lira do të kisha mundur të vendosja qetësinë në shtëpi dhe të bëja të rilindë dashuria e vrarë padrejtësisht nga vejusha Peskatore? (RAMJA, VI, 67)

- Me njëmbëdhjetë mijë lireta a mund të rehatoj shtëpinë e të ringjall dashurinë, të cilën e vrau ndyrësisht që sapo lindte vejusha Peskatore? (CANKO, VI, 64)

$>$ Quando trafelato, in uno stato miserando, dopo aver girato tutte le farmacie, rincasai, disperato e furibondo, la prima bambina era già nata; si stentava a far venir l'altra alla luce.

- Kur i rraskapitur e në gjendje për tu mishëruar, pasi isha sjellë në të gjitha farmacitë, u ktheva në shtëpi i dëshpëruar e i çoroditur, vajza e parë kishte lindur e përpiqej të dilte në jetë tjetra. (RAMJA, V, 50)

- Kur i strapacuar, në një gjendje të vajtueshme, pasi s'kam lënë farmaci pa shkelur, futem në shtëpi, i dëshpëruar dhe i hakërryer, kërthia e parë ka dalë në dritë; po përpiqet të lindë edhe tjetra. (CANKO, V, 48)

Questi latinismi sono parole che si usano nello stile letterario, dunque appartengono ad un registro particolare. Come dice Bruno Osimo (2007: 48-49): «Analizzando la lingua di un testo si notano casi di marcatezze lessicali, cioè quando un termine che appartiene ad un registro particolare viene inserito in un contesto ad esso estraneo. Nella traduzione si dovrebbe cercare di riprodurre le marcatezze lessicali» Si nota invece, che nella traduzione sono stati usati equivalenti, che non appartengono esclusivamente allo stile letterario. Abbiamo a che fare con una perdita inevitabile del processo della traduzione. Qualsiasi lingua ha le sue peculiarità dell'origine e dell'evoluzione, intrecciate dalle particolarità culturali di ogni nazione. Impossibile che venga creato lo stesso effetto che ha voluto creare l'autore della lingua originale del romanzo, perché l'italiano e l'albanese non condividono lo stesso contesto storico di evoluzione.

\subsection{Esempi tratti dal romanzo Uno, nessuno e centomila.}

In questo romanzo si nota la presenza di latinismi integrati.

> Mi volevano proprio interdire, denunziandomi come alterato di mente. Dida le aveva annunziato che già erano state raccolte e ordinate tutte le prove, da Firbo, da Quantorzo, da suo padre e da lei stessa, per dimostrare la mia lampante alterazione mentale.

- Donin pikërisht të më hiqnin të drejtën e profesionit, duke më paditur si të lajthitur nga trutë. Dida i kishte thënë që tashmë ishin mbledhur dhe porositur të gjitha provat, nga Firbo, nga Kuantorxo, nga babai i saj dhe nga ajo vetë, për të treguar tjetërsimin tim të qartë mendor. (ÇELA, Libri i shatë, IV, 152)

- Donin të më hiqnin të drejtën, duke më denoncuar si të luajtur mendsh. Dida e kishte lajmëruar se tashmë ishin mbledhur e sistemuar të gjitha provat, nga Firboja, nga Kuantorci, nga i ati e nga ajo vetë, për të treguar çrregullimin tim të qartë mendor. (VUKAJ, Libri i shtatë, IV, 171)

$>$ [...] tant'altre cose m'erano state fatte, date da altri, a cui effettivamente io non avevo mai pensato, mai dato immagine, l'immagine strana, nemica, con cui mi s'avventavano adesso.

- [...] plot gjëra të tjera m'i kishin bërë, m'i kishin dhënë të tjerët, për të cilët në fakt kurrë nuk kisha menduar, kurrë nuk u kisha dhënë një shëmbëlltyrë, shëmbëlltyrën e çuditshme, armike, me të cilën më turreshin tani. (ÇELA, Libri i tretë, II, 60)

- [...] gjithë ato gjëra të tjera më kishin qenë bërë, dhënë prej të tjerëve, për të cilat në të vërtetë unë nuk kisha menduar kurrë, s'kisha dhënë kurrë përfytyrimin e çuditshëm, armik, me të cilin më vështronin tani. (VUKAJ, Libri i tretë, II, 68)

$>$ Ricostruisce, ricostruisce, bestiolina pervicace.

- Rindërton, rindërton, kafshëz kokëfortë. (ÇELA, Libri i dytë, XI, 48)

- Rindërton, rindërton, kafshëza kokëfortë. (VUKAJ, Libri i dytë, XI, 56)

> Bisognava però, perché questa confessione non fosse pericolosa, che io dimostrassi nello stesso tempo cosí viva e urgente per la mia anima la necessità d'un eroico ravvedimento, da ridare a lui l'animo e la forza di chiedere agli altri il sacrifizio dei proprii interessi. 
- Mirëpo, që ky rrëfim të mos qe i rrezikshëm, duhej që, në të njëjtën kohë, të tregoja nevojën aq të gjallë e të menjëhershme në shpirtin tim të një pendese historike, çka do t'i jepte atij zemër dhe forcë t'u kërkonte të tjerëve sakrifikimin e interesave të tyre. (ÇELA, Libri i tetë, III, 175)

- Por duhej, në mënyrë që ky rrëfim të mos ishte i rrezikshëm, që unë ta tregoja në të njëjtën kohë kaq të gjallë e urgjente për shpirtin tim, nevojën e një pendimi heroik, për t'i ridhënë atij zemrën dhe forcën që t'u kërkonte të tjerëve sakrifikimin e interesave të tyre. (VUKAJ, Libri i tetë, III, 197)

$>$ E rideva, rideva con un lustro di lagrime negli occhi spiritati, mentre gli volavano di qua e di là, lingueggiando come fiamme, le lunghe ciocche dei capelli rossicci.

- Dhe qeshte, qeshte me një shkëlqim lotësh te sytë e gjallëruar, teksa i fluturonin andej e këndej, si gjuhë flake, cullufet e gjata të flokëve të kuqerrema. (ÇELA, Libri i shtatë, VII, 159)

- Dhe qeshte, qeshte me një shkëlqim lotësh në sytë e çakërdisur, ndërsa i fluturonin andej e këtej, duke u përpëlitur si flakë, cullufet e flokëve të kuqërremtë. (VUKAJ, Libri i shtatë, VII, 178)

> Tanti erano pronti a farne testimonianza finanche quel Turolla che avevo difeso contro Firbo e tutti i commessi della banca; finanche Marco di Dio a cui avevo fatto donazione d'una casa.

- Shumë njerëz ishin të gatshëm të dëshmonin për këtë; deri edhe ai Turola që e kisha mbrojtur kundër Firbos dhe të gjithë nëpunësve të bankës; deri edhe Marko di Dio, të cilit i kisha dhuruar një shtëpi. (ÇELA, Libri i shatë, IV, 152)

- Shumë ishin gati të dëshmonin për këtë; madje edhe ai Turola, që e kisha mbrojtur kundër Firbos, e të gjithë punonjësit e bankës; deri edhe Marku i Zotit, të cilit i kisha bërë dhurimin e një shtëpie. (VUKAJ, Libri i shtatë, IV, 171)

$>$ [...] non però quanto a realtà, vi prego di credere perché non meno reale di questo Giulio Cesare imperiale era quel lezioso fastidioso tutto raso e discinto e infedelissimo di sua moglie Calpurnia: o quello impudicissimo di Nicomede re di Bitinia.

- [...] ju lutem të më besoni, sepse jo më pak i vërtetë nga ky Jul Qezar madhështor ishte ai nazemadh i bezdisshëm, gjithë i rruar e gjysmë i zhveshur dhe aspak besnik ndaj së shoqes, Kalpurnias: apo tejet $\mathrm{i}$ paturpshëm i Nikomedit, mbret i Bitinias. (ÇELA, Libri i katërt, I, 81)

- [...] ju lutem besojeni, sepse jo më pak real se ky Jul Qezar perandorak ishte ai nazemadhi i bezdisshëm, krejt i rruar, gjysmë i zhveshur dhe aspak besnik ndaj gruas së tij, Kalpurnia: apo aq shumë i pacipë me Nikomedin, mbret i Bitinisë. (VUKAJ, Libri i katërt, I, 93)

$>$ A Richieri si era avvezzi al fasto, alle maniere gioconde e cordiali, alla copiosa munificenza del suo predecessore, il defunto Eccellentissimo Monsignor Vivaldi;

- Në Rikieri ishin mësuar me salltanetin, me sjelljet e hareshme e të përzemërta, me bujarinë e bollshme të pararendësit të tij, të ndjerit Fort të Nderuar Imzot Vivaldit; (ÇELA, Libri i shtatë, IV, 156)

- Në Rikieri ishin mësuar me salltanetin, me sjelljet gazmore e të përzemërta, e me një bujari të bollshme të paraardhësit të tij, të të ndjerit Monsinjor Vivaldi; (VUKAJ, Libri i shtatë, IV, 176)

Anche in questo romanzo come in quello precedente i latinismi usati da Pirandello sono parole che appartengono allo stile letterario. Si nota che nella traduzione sono stati usati equivalenti, che appartengono all'uso comune della lingua, perciò il lettore della traduzione non coglie questo tratto distintivo della lingua di Pirandello.

\subsection{Esempi tratti dal romanzo L'Esclusa.}

\subsubsection{Latinismi non integrati}

$>\mathrm{NIHIL}-\mathrm{MIHI}-\mathrm{CONSCIO}$

- Nihil - Mihi - Conscio (Pjesa e parë, Kapitulli i dytë, 25)

$>$ Ora pro nobis.

- Ora pro nobis. (Pjesa e parë, Kapitulli i dytë, 25)

> E al capezzale l'Ecce homo d'avorio, riparato da una lastra concava dentro la cornice ovale, nera; l'Ecce homo che una volta aveva chinato in segno d'assentimento il capo incoronato di spine a lei e a Maria accorse una dopo l'altra a supplicarlo per la madre colta da improvviso malore.

- Dhe në krye të shtratit Ecce Homo3-ja prej fildishi, e mbajtur nga një pllakë e lugët brenda kornizës së zezë vezake; Ecce Homo që njëherë u pati përkulur në shenjë pranimi kokën e kurorëzuar me gjëmba asaj dhe Marijes që kishin rendur njëra pas tjetrës t'i luteshin për nënën që ishte goditur befas nga një sëmundje e 
papritur. (Pjesa e parë, Kapitulli i katërt, 35)

3-ECCE HOMO latinisht - [Ja njeriu] shqiptuar nga Pilati

$>$ Pompeo Emanuele Mormoni lo chiamava l'istrice e, da imperatore romano, lo avrebbe condannato ad purgationem cloacarum.

- Pompeo Emanuele Mormoni e quante «iriqi» dhe nga perandori romak do të ishte dënuar ad purgationem cloacarum ${ }^{7}$. (Pjesa e dytë, Kapitulli i dytë, 128)

7- AD PURGATIONEM CLOACARUM lat. - me pastrim gjirizesh.

Per quanto riguarda la strategia traduttiva, si può dire che il traduttore ha seguito la strategia della ripetizione per i segmenti in latino che appaiono in frasi indipendenti. Mentre per le espressioni intercalate all'interno delle frasi, oltre alla ripetizione, viene aggiunta anche una glossa extratestuale che si trova in un piccolo vocabolario alla fine del libro in cui si da il significato del segmento testuale.

\subsubsection{Latinismi integrati}

> Potrei entrare, anche adesso; farmi annunziare, vederla e parlarle.

- Mund të hyja edhe tani, ta lajmëroja, ta shihja e t'i flisja. (Pjesa e dytë, Kapitulli i shtatë, 154)

$>$ Venne finalmente, inavvertito dalla morente, il Viatico.

Erdhi më në fund, i pa vënë re nga ajo që po vdiste, kungimi i fundit. (Pjesa e dytë, Kapitulli i katërmbëdhjetë,

> In mezzo al bujo androne, l'Ajala, con le mani intrecciate dietro la nuca, le braccia strette intorno alla testa, s'era messo a guardare la grande porta a vetri, in fondo, cieca nel blando chiaror lunare.

- Në mes të korridorit të errët, Ajala, me duar të kryqëzuara pas zverkut, krahët shtrënguar rreth kokës, po vështronte portën e madhe me xhama, në fund, të verbër në ndriçimin e ëmbël të hënës. (Pjesa e parë, Kapitulli i tretë, 29)

> Fatti in fretta i preparativi della partenza, appena in viaggio, aveva provato un subitaneo sollievo quasi insperato, come se le nebbie gli si fossero a un tratto diradate attorno.

- Pasi kishte bërë me nxitim përgatitjet e nisjes, sapo ishte në udhëtim, kishte provuar një lehtësim të menjëhershëm, gati të pashpresuar, a thua se mjegullat përreth i qenë davaritur. (Pjesa e dytë, Kapitulli i shtatë, 153)

$>$ Sapeva, sapeva d'essere inviso a tutti, cominciando dagli stessi suoi figli.

- E dinte, e dinte se ishte i urryer prej të gjithëve, duke filluar nga vetë bijtë e tij. (Pjesa e parë, Kapitulli i dhjetë, 76)

> Improvvisamente, barcollando, urlando, con le braccia levate, furibonda dagli spasimi e dalla paura, irruppe in quella stanza Marta, discinta, scarmigliata, inseguita dalla madre e dalle donne assistenti.

- Befas, duke u lëkundur, duke ulëritur, me krahët e ngritur lart, e tërbuar nga dhimbjet dhe nga frika, vërshoi në atë dhomë Marta gjysmë e zhveshur, flokëshprishur, e ndjekur nga e ëma dhe nga gratë asistente. (Pjesa e parë, Kapitulli i gjashtë, 49)

$>$...e lui, che non si sentiva il coraggio di esporle il miserando stato delle cose, s'adoperava in tutti i modi perché, ogni mese, non mancasse almeno il denaro per lei.

- Dhe ai, që nuk gjente kurajën t'i paraqiste gjendjen e vajtueshme të gjërave, orvatej me të gjitha mënyrat që çdo muaj, të paktën asaj, të mos i mungonin paratë. (Pjesa e parë, Kapitulli i shtatë, 58)

$>$ Che dici? - domandò con viva ansia il figlio, curvandosi vie più su lei.

- Çfarë thua? - pyeti me një ankth të fortë i biri, duke u përkulur edhe më shumë mbi të. (Pjesa e dytë, Kapitulli i pesëmbëdhjetë, 208)

> Né l'uno né l'altro potevano seriamente ingelosirsi del Falcone, il quale faceva loro finanche pietà, via!

- As njëri, as tjetri nuk mund të ishin vërtet xhelozë për Falkonen, të cilin madje e mëshironin, dhe kaq! (Pjesa e dytë, Kapitulli i dytë, 131)

Questi latinismi come abbiamo già detto si usano nello stile letterario. Si nota che nella traduzione a volte sono state usate parole dello stile letterario albanese (i vajtueshëm, madje ecc.), mentre in altri casi sono stati usati (come succede anche per gli altri romanzi) equivalenti del linguaggio comune. 


\section{Conclusioni}

Per concludere possiamo dire che tradurre è un lavoro molto difficile anche in quei casi in cui apparentemente non sussistono difficoltà. Nel nostro studio abbiamo visto come la lingua di Pirandello, apparentemente facile, induce il traduttore in difficoltà e richiede che elabori la sua strategia traduttiva. Trovandosi di fronte ai latinismi usati da Pirandello i traduttori hanno elaborato quasi quasi la stessa strategia: ripetizione per i latinismi non integrati dando nella maggior parte dei casi il loro significato con delle glosse extratestuali; e, traduzione nella lingua albanese per i latinismi integrati optando a conservare, però, soltanto il piano semantico della traduzione tralasciando quello stilistico, poiché gli equivalenti usati nella maggior parte dei casi sono termini di uso comune e non appartengono alla lingua letteraria 0 dotta. Questa peculiarità della lingua di Pirandello, queste marcattezze del suo linguaggio non vengono date nella lingua albanese dando luogo così a quelle che chiamiamo perdite irrecuperabili in una traduzione.

\section{References}

Fonti Primarie

Pirandello, L. (1999). Marta Ajala, tradotto da Zija Vukaj, Tirana: Elite. (2000). Tri herë vetvrasës, tradotto da Sabah Ramja, Shkodër: Helena Kadare. (2001). Një, asnjë dhe njëqindmijë, tradotto da Zija Vukaj, Tirana: Elite. , (2002). Një, asnjë dhe njëqindmijë, tradotto da Dritan Çela, Elbasan: Sejko. , (2002). I ndjeri Matia Paskal, tradotto da Violanda Canko, Tirana: Elena Gjika.

\section{Fonti Secondarie}

Carbonell i Cortés O., (1999). Traducción y cultura: de la ideología al texto. Madrid: Ediciones Colegio de España.

Catford, J. C, (1965). A Linguistic Theory of Translation, An Essay in Applied Linguistics, Language and language learning. London: Oxford University Press.

Crepaldi, C. (a cura di) (2002). Sintesi II fu Mattia Pascal di Luigi Pirandello. Introduzione all'opera. Riassunto e analisi. Approfondimenti. Vita e opere dell'autore. Milano: Avallardi.

Dardano, M. \& Trifone, P. (1997). La nuova grammatica della lingua italiana, Bologna, Zanichelli.

Filippo, P. (1968). II dialetto siciliano nella lingua di Pirandello. In Pirandello e la sua lingua. Cappelli Editore.

Gjinari, J. \& Shkurtaj. Gj. (2009). Dialektologjia, Tirana: shblu.

Lauretta, E. (1952). Come leggere «ll fu Mattia Pascal». Milano: Mursia.

Lauretta, E. (1993). II fu Mattia Pascal, Testo integrale, schede storiche e letterarie, note al testo. Vimercate: Meravigli Editrice.

Lefevere, A. (1992). Translating Literature: Practice and Theory in a Comparative Literature Context. New York: The Modern Language Association of America.

Lefevere, A. (1997). Traducción, reescritura y la manipulación del canon leterario. Ma Carmen Africa Vidal e Román Alvarez (traduttori). Salamanca: Ediciones Colegio de España (Translation, Rewriting and the Manipulation of Literary Fame, Londër: Routledge, 1992).

Momigliano, A. (1960). Storia della letteratura italiana. Principato.

Navarro Blanco, A. (1990). La lengua y el estilo de Luigi Pirandello, tesis doctoral.

Osimo, B. (2004). Manuale del traduttore, Milano: Hoepli.

Osimo, B. (2007). La traduzione saggistica dall'inglese, Milano: Hoepli.

Papa, M. (2011). Sulla traduzione nella lingua albanese delle opere del nobelista italiano Luigi Pirandello, tesi dottorale.

Papa, M. \& Xhamani, L. (2013). Difficulties in translating the language of Pirandello's novels - articolo pubblicato sulla rivista «Academic Journal of Interdisciplinary Studies» Vol. 2, No. 8, MCSER Publishing Rome, pp. 231-240, Roma, Italia, ISSN 2281-3993.

Pirandello, L. (1993). II fu Mattia Pascal. Testo integrale. Schede storiche e letterarie. Note al testo. Milano: Meravigli Editrice.

Rabadán, R. (1994). Traducción, intertextualitad y manipulación. in Amparo Hurtado Albir (ed.) Estudis Sobre la traducció. Castelló: Universitat Jaume I, 129-39. 